\title{
Didacticiel d'Instrumentation Virtuel à Coût Réduit pour l'Acquisition et le Traitement des Signaux ECG
}

\author{
G. TCHIMMOUE ${ }^{12}$, J. KAMDEM ${ }^{2}$, A. S. NKO'O ${ }^{3}$ \\ ${ }^{1}$ Laboratoire d'Automatique et d'Informatique Appliquée (LAIA), Département de Génie Electrique, \\ Université de Dschang, B.P. 134 Bandjoun, Cameroun, Email: tchimmoue@ yahoo.fr. \\ ${ }^{2}$ Laboratoire d'Electronique et de Traitement du Signal (LETS), Ecole Nationale Supérieure Polytechnique, \\ Université de Yaoundé I, BP 8390 Yaoundé, Cameroun, Email: kamdem_kouokam@yahoo.fr. \\ ${ }^{3}$ Faculté de Médecine et des Sciences Biomédicales, \\ Université de Yaoundé I, BP 1364 Yaoundé, Cameroun, Email: snkooamvene@ yahoo.fr
}

\section{RESUME:}

Cet article présente un didacticiel d'instrumentation virtuel à coût réduit pour l'acquisition et le traitement des signaux ECG. Il s'adresse aux étudiants des filières I.M.B. (Instrumentation et Maintenance Biomédicale) et Génie électrique ou E.E.A. (Electronique Electrotechnique - Automatique) en leurs permettant, lors de séances de Travaux Pratiques, d'étudier concrètement les différentes parties qui entrent dans la constitution d'une chaine d'acquisition par ordinateur, et de comprendre les notions clés de l'instrumentation en général et de l'instrumentation biomédical en particulier. La partie matérielle du prototype est constituée d'un générateur de signaux ECG puis de trois étages amplificateur d'instrumentation et filtres analogiques suivi, pour la numérisation, d'un microcontrôleur doté d'un convertisseur A/N et relié directement au connecteur DB 25 du port LPT d'un PC. Le logiciel prévu pour piloter la carte d'acquisition a été développé en Visual Basic. L'intérêt de ce didacticiel est fondé sur le coût réduit, la facilité d'utilisation et la possibilité de sauvegarder tous les résultats obtenus sous forme d'un fichier d'extension . $M$, ce qui permet d'avoir accès aux outils de traitements numériques supplémentaires que propose MATLAB.

Mots clés : Didacticiel, ECG, amplificateur d'instrumentation, microcontrôleur, port parallèle, filtre numérique, MATLAB.

\section{Introduction}

L'acquisition et le traitement numérique des signaux en général représentent une partie importante dans le programme de formation des étudiants des filières technologiques. Les kits de développements commerciaux tels que le Medical Development Kit conçue autour du VC5505 de Texas Instruments seraient une bonne alternative pour les enseignements pratiques de ces matières, cependant ces kits sont des systèmes hautement intégrés grâce aux technologies FPGA et DSP [1-2-3] et sont de ce fait difficiles à maintenir en cas de panne et onéreux notamment pour les institutions de formation des pays en voie de développement. Cet article présente un didacticiel d'instrumentation virtuelle peu couteux, conçu et réalisé pour les travaux pratiques dans le domaine de l'acquisition et du traitement numérique par ordinateur des signaux ECG. L'intérêt pédagogique de cette chaine est double : 
- Permettre de montrer concrètement aux étudiants, grâce a la modularité de la maquette, les différentes parties qui entrent dans la conception d'une chaine d'acquisition par ordinateur complète en allant du signal à mesurer jusqu'à l'organe d'acquisition numérique ;

- Permettre aux étudiants, par l'acquisition et le traitement des signaux ECG, de visualiser et comprendre les notions clés de l'instrumentation tels que le bruit, l'amplification, le filtrage analogique et numérique, l'échantillonnage, la représentation spectrale.

Apres avoir présenté en seconde partie l'interface matérielle de la chaine d'acquisition, nous aurons en troisième partie le développement de l'interface logiciel qui sera chargé de piloter la carte d'acquisition. Les différentes étapes de manipulation de la maquette lors d'une séance de travaux pratiques achèveront ce document.

\section{Interface matériel}

Le schéma synoptique du kit proposé est représenté à la Figure1. Il est composé de quarte principales parties: Le générateur de signaux ECG, les amplificateurs d'instrumentation auxquels sont associés les filtres, la carte d'acquisition et un ordinateur PC doté d'un port LPT et contenant le logiciel d'instrumentation virtuelle. La réalisation et le choix des composants des cartes électroniques doivent satisfaire à la triple contrainte de facilité de maintenance et de programmation, de coût réduit et de disponibilité sur le marché local.

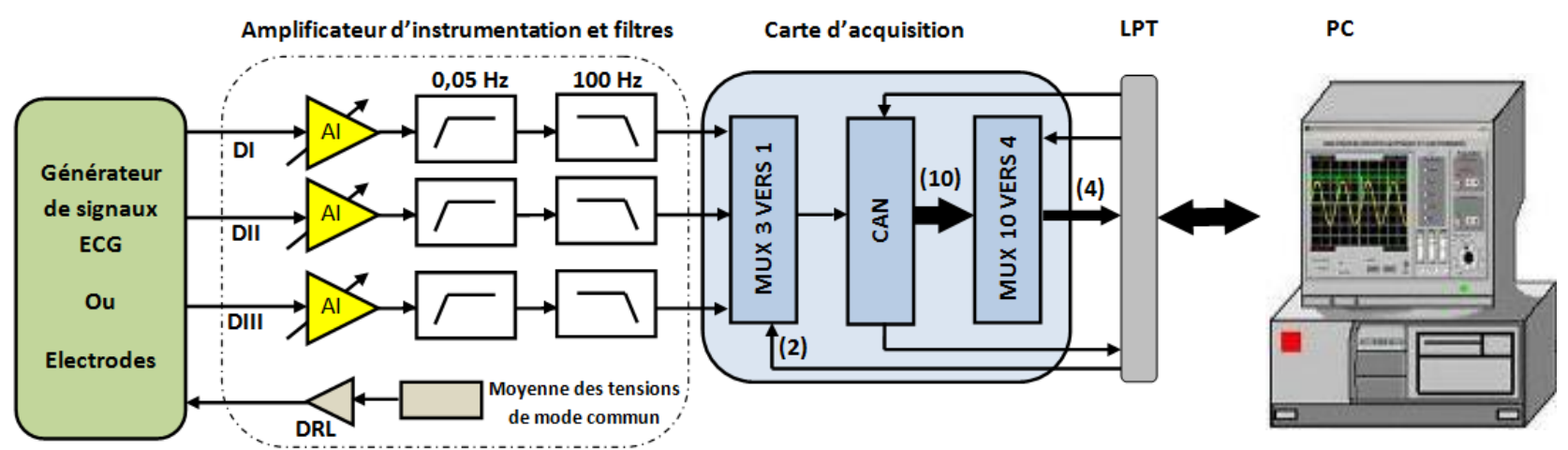

Figure 1 : Schéma synoptique de la chaine d'acquisition

\subsection{Générateur de signaux ECG}

Les signaux d'entrée de la chaine sont fournis par le générateur de signaux ECG. Le circuit électrique correspondant est donné sur la figure 2 et est conçu autour du microcontrôleur PIC 16F84A de MICROCHIP. Le principe de génération est le suivant : Une période d'un signal ECG échantillonné à une fréquence de $255 \mathrm{~Hz}$ et numérisé à 8 bits est rangé dans un tableau et stocké dans la mémoire FLASH du microcontrôleur. Ce dernier envoie en boucle et à une vitesse de $255 \mathrm{~Hz}$ les données du tableau au convertisseur numérique analogique DAC0808. En utilisant ce même principe et en fonction de l'état des interrupteurs, le microcontrôleur peu générer à la sortie du DAC trois signaux différents:

- un signal ECG typique ; 
- un signal ECG bruité par le $50 \mathrm{~Hz}$ de l'alimentation ;

- un signal sinusoïdal de $10 \mathrm{~Hz}$.

Le pont diviseur de tension à la sortie de l'amplificateur opérationnel (U3:A) permet de ramener aux bornes de la résistance R6 les signaux à l'ordre du $\mathrm{mV}$ ainsi, les signaux ECG sont à $3 \mathrm{mV}$ crête à crête d'amplitude maximale et le signal sinusoïdal à 3,5 mV crête à crête. Ce dernier signal est utilisé pour régler le gain des amplificateurs d'instrumentation. Notons que ce montage ne nécessite pas de mémoire EEPROM externe pour le stockage des tables de données numérisées contrairement au générateur de signaux ECG étudié par Martinez et al. [4], ce qui permet de réduire le nombre de composants et par conséquent le coût de la réalisation.

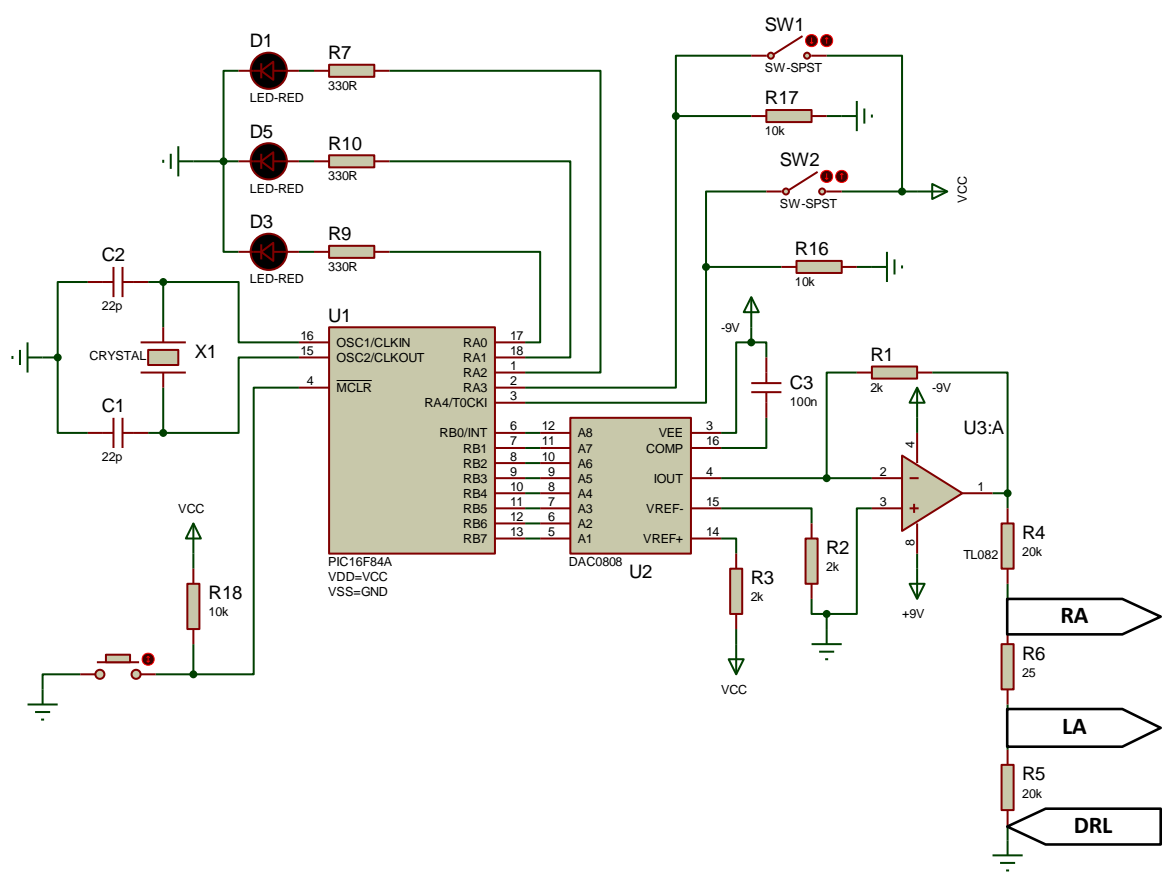

Figure 2 : Circuit électrique du générateur d'ECG conçue autour du PIC 16F84A et du DAC 0808.

\subsection{Les amplificateurs d'instrumentation et les filtres}

Les signaux ECG qui sont fournis par le générateur d'ECG synthétique sont destinés à attaquer les étages amplificateurs d'instrumentation à trois amplificateurs opérationnels. Le schéma de la figure $3 a$ illustre un des trois étages amplificateur d'instrumentation, les amplificateurs opérationnels utilisés sont des TL082. Grâce à leurs étages d'entrée à technologie JFET ils permettent d'avoir une impédance d'entrée importante $\left(10^{12} \Omega\right)$ et un taux de rejection en mode commun élevé $(100 \mathrm{~dB})$. La résistance de gain est un ajustable et permet le réglage du gain de 100 à 1800. A la suite des étages amplificateurs, se trouvent à la figure $3 \mathrm{~b}$ trois étages de filtres de second ordre Sallen-Key passe-haut et un autre passe-bas de gain unité. Pour ne laisser passer que les harmoniques du signal cardiaque, les fréquences de coupures respectives des filtres sont fixées à $0,05 \mathrm{~Hz}$ et $101 \mathrm{~Hz}$. 

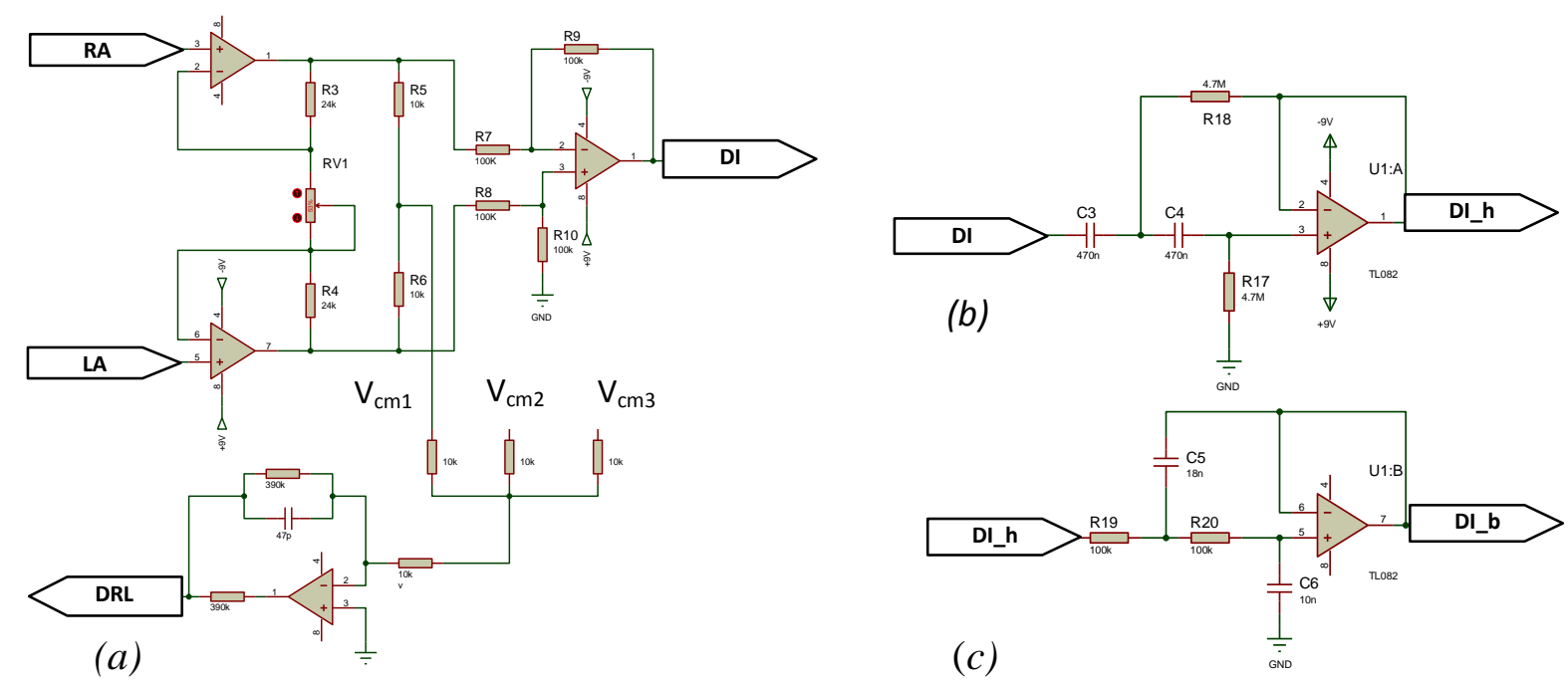

Figure 3 : Un des trois étages amplificateur d'instrumentation à trois ampli op (a), de filtre passe haut (b) et passe bas de second ordre (c).

\subsection{La carte d'acquisition}

La carte d'acquisition dont le circuit électrique est représenté à la figure 4 permet de numériser les signaux issus des filtres avant de les envoyer via le port LPT au PC. Elle est conçue autour du microcontrôleur PIC 16F877 de MICROCHIP dont on exploite principalement la fonction convertisseur analogique numérique. Le choix de ce composant a été fait sur la base des considérations pratiques telles que:

- Un coût réduit et une grande disponibilité sur le marché local ;

- La présence d'un ADC de 10 bits avec 3 entrées analogiques ;

- L'utilisation des outils de développement et de programmation variés et disponibles ;

- La facilité de connexion au PC via sont port LPT sans nécessiter un circuit d'adaptation supplémentaire.

Les amplificateurs opérationnels montés en suiveur offrent à la carte d'acquisition une grande impédance d'entrée tandis que les réseaux de résistances branchées à la suite constituent un circuit de décalage pour permettre au microcontrôleur de numériser les tensions négatives. Une Diode Zener $(V z=3,1 \mathrm{~V})$ limite les signaux analogiques aux entrées de la carte d'acquisition entre $+3 \mathrm{~V}$ et $-3 \mathrm{~V}$.

Le programme implanté dans la mémoire du microcontrôleur a été développé en $\mathrm{C}$ à l'aide du compilateur CCS (Custom Computer Services). En fonction de l'état des broches de commande Start et Mux ce programme gère les conversions analogique numériques et envoie les résultats obtenu en trois quartets de bits sur les 4 bits de poids faible du port D configuré 
en sortie. Ce transfert de quartet de bit du microcontrôleur vers le PC se fait dès la fin de la conversion $(E O C=1)$ et de façon séquentielle en fonction de l'état de la broche Mux.

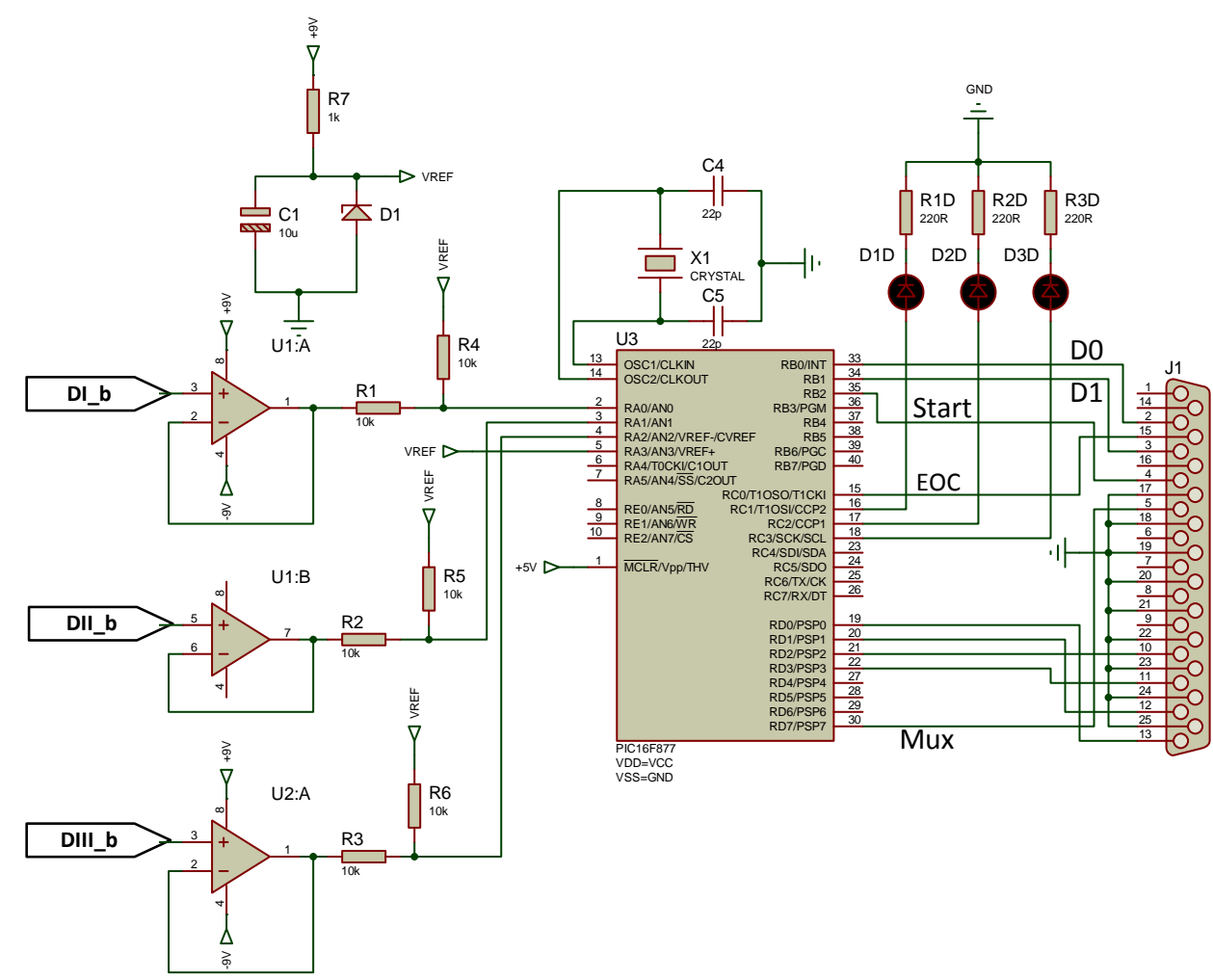

(a)

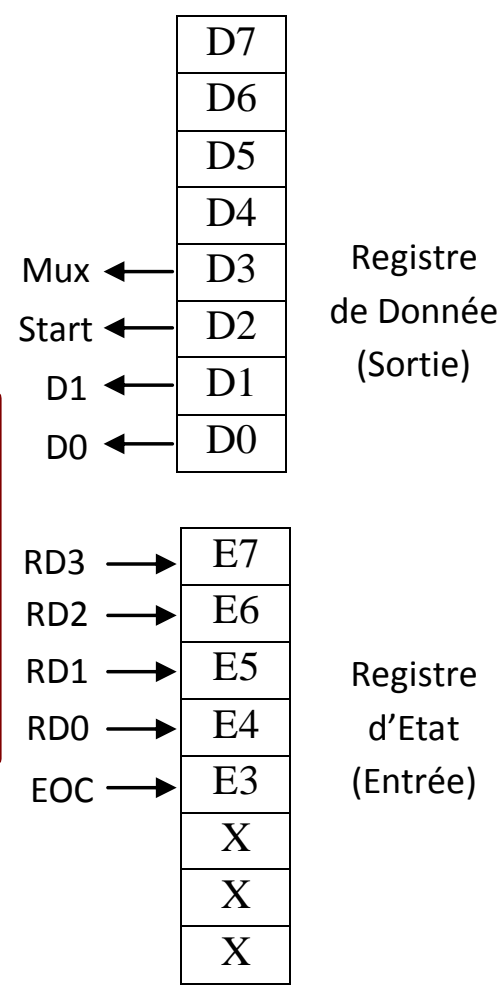

(b)

Figure 4 : (a) Circuit électrique de la carte d'acquisition conçue autour du PIC $16 F 877$ et (b) assignation des $E / S$ du port $L P T$

Le tableau 1 montre l'état des LED témoins et l'ordre de conversion des tensions aux trois entrées analogiques $\mathrm{AN} 0, \mathrm{AN} 1$, et $\mathrm{AN} 2$ en fonction de l'état des broches D0 et D1 pilotées par le programme du PC. Les organigrammes des programmes qui gèrent cette conversion coté microcontrôleur et coté PC sont présentés dans la partie développement de l'interface logiciel.

Tableau 1 : Sélection des entrées analogiques et Etat des LED témoins en fonction de DO et D1 piloté par le PC

\begin{tabular}{|c|c|c|c|}
\hline D1 & D0 & LED allumée & Entrées analogique \\
\hline 0 & 0 & D1D & AN0 \\
\hline 0 & 1 & D2D & AN1 \\
\hline 1 & 0 & D3D & AN2 \\
\hline 1 & 1 & D1D, D2D, D3D & AN0+AN1+AN2 \\
\hline
\end{tabular}




\subsection{Prise de vue de la maquette}

La figure 5 présente une photographie de la chaine d'acquisition complète. Le typon des circuits imprimés a été conçu avec le logiciel de CAO Proteus.

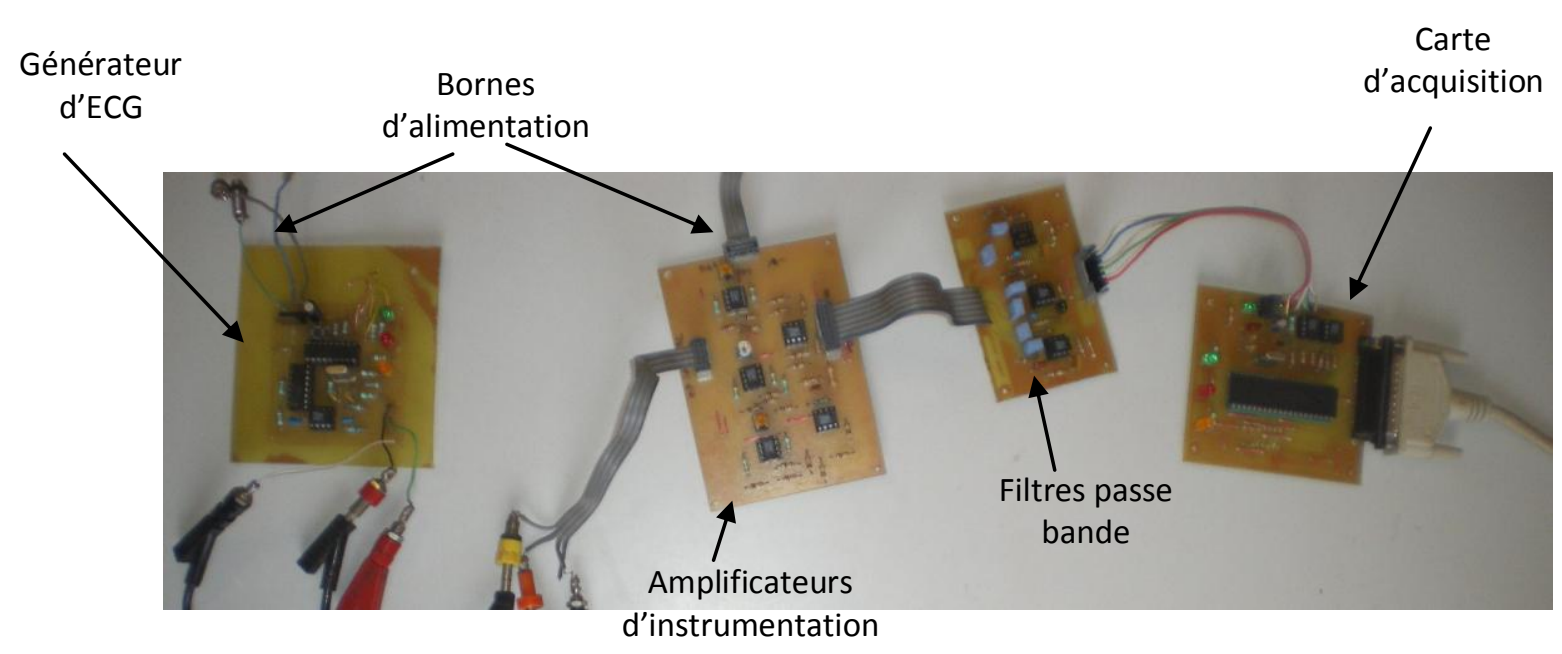

Figure 5 : Photographie de la chaine d'acquisition.

\section{Développement de l'interface logiciel}

Le logiciel implanté dans le PC et chargé de piloter la carte d'acquisition a été développé sous Visual Basic 6 de Microsoft. Pour accéder en écriture et en lecture aux registres $\mathrm{du}$ port LPT dans l'environnement Windows XP, la librairie dynamique Inpout32.DLL a été utilisée. Cette librairie est téléchargeable gratuitement sur internet [int1] et quelques exemples de code source en Visual basic expliquant son utilisation sont consultable dans plusieurs documents [5]. L'interface logicielle est conçue avec le souci de le rendre le plus intuitif possible.

\subsection{Fonctionnalités du logiciel}

L'interface logicielle développée propose deux principaux modes de fonctionnement : le mode visualisation et le mode Filtrage et TFD.

- Sous le mode Visualisation il est possible :

- d'estimer la fréquence d'échantillonnage maximale du PC qui est prise comme référence pour les calculs des nombres d'échantillons à afficher ;

- de régler le gain des amplificateurs d'instrumentation en jouant sur les résistances de gain ajustables ;

- d'afficher en temps réel l'allure de la tension présente sur l'une des trois entrées ou sur les trois entrées simultanément ;

- de régler, comme pour un oscilloscope, la base des temps ou la base des tensions pour avoir l'affichage la plus adaptées ; 
- de sauvegarder les données dans un fichier d'extension.$M$ pour traitement à l'aide de MATLAB ou pour archivage.

- Sous le mode Filtrage et TFD on peut :

- suivant le nombre d'échantillon et de la fréquence d'échantillonnage choisi, afficher l'allure d'une seule des trois entrées ;

- traiter le signal acquis à l'aide d'un filtre numérique à Réponse Impulsionnelle Infini d'ordre 3 et dont les coefficients sont saisis dans des zones de texte prévue à cet effet ;

- représenter l'allure du signal filtré ;

- calculer et représenter l'amplitude de la Transformée de Fourier Discrète des signaux acquis et filtré.

La figure 6 représente une copie d'écran de l'interface logicielle à son démarrage.

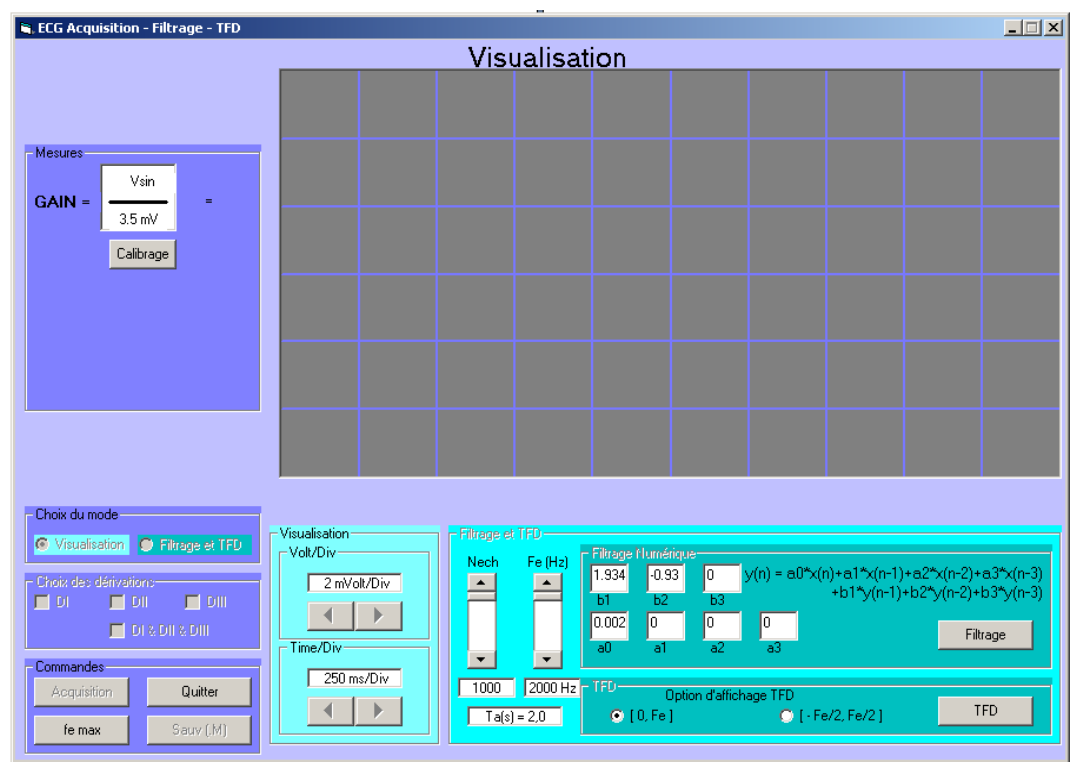

Figure 6 : Logiciel d'instrumentation virtuel à son lancement.

\subsection{Organigrammes et programmes des différents modules constitutifs du logiciel.}

Le logiciel d'instrumentation virtuel proposé a été conçu autour des principaux modules ou sous programmes tel que l'acquisition, le filtrage numérique récursif, la Transformée de Fourier Discrète et la sauvegarde de données dans un fichier d'extension.M.

\subsubsection{Le module acquisition}

Ce module permet au PC de se synchroniser avec la carte d'acquisition et de récupérer les données issues du convertisseur analogique numérique. La figure ci-dessous illustre l'organigramme des algorithmes des programmes contenu dans le microcontrôleur et celui développé en Visual basic pour le PC. 

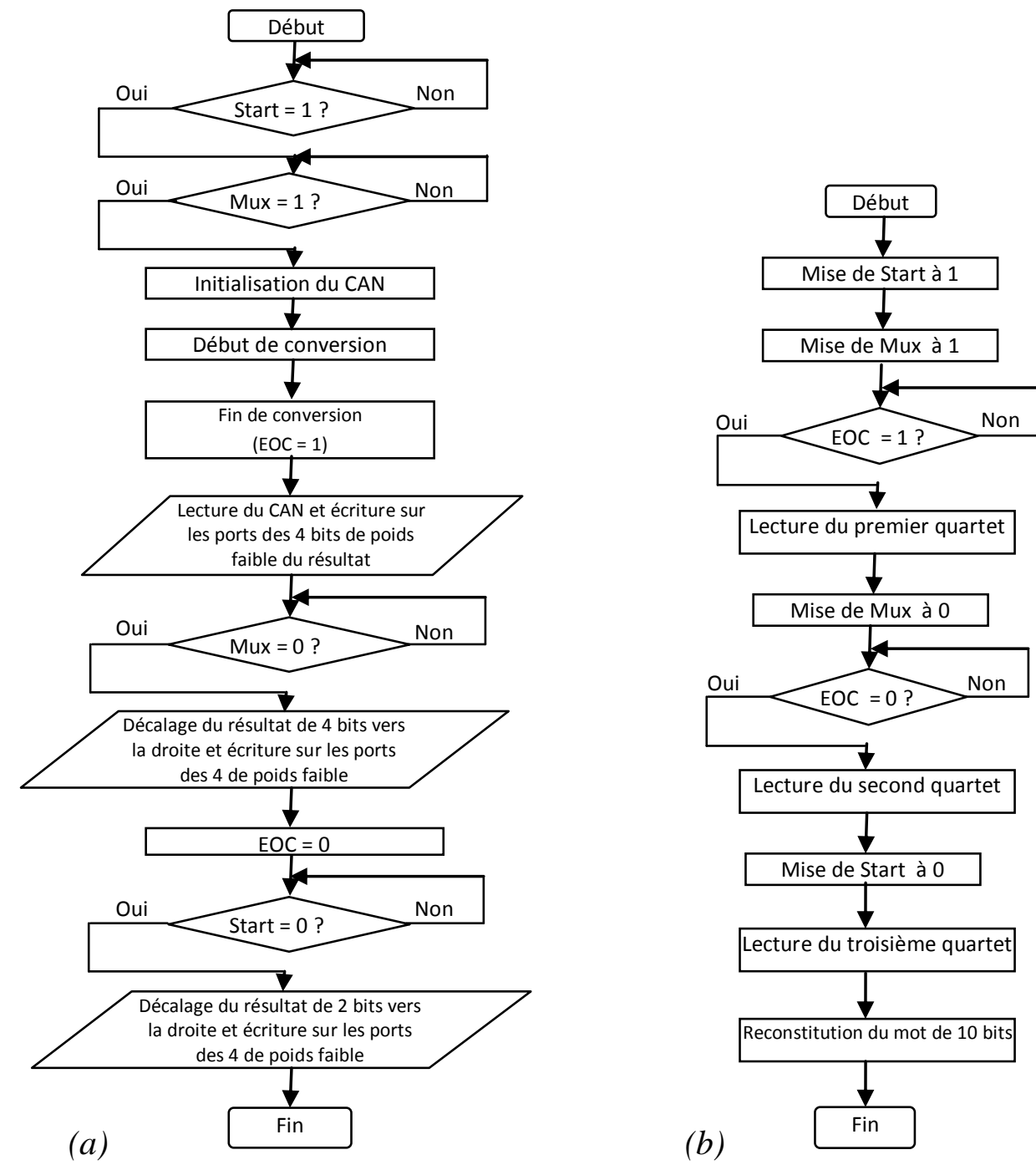

Figure 7. Organigramme des algorithmes d'acquisition : (a) algorithme de conversion implanté dans le microcontrôleur, et (b) algorithme d'acquisition implanté dans le PC.

\subsubsection{Le module filtrage numérique à RII et de la TFD.}

L'organigramme de la figure 8 présente l'algorithme du filtrage numérique dont l'équation aux différences est donnée par l'équation (1) et celui de la Transformée de Fourier Discrète d'un signal tronqué sur $\mathrm{N}$ échantillons avec pour expression la relation (2)[6].

$$
\begin{array}{r}
\mathrm{y}(\mathrm{n})=\mathrm{a} 0 * \mathrm{x}(\mathrm{n})+\mathrm{a} 1 * \mathrm{x}(\mathrm{n}-1)+\mathrm{a} 2 * \mathrm{x}(\mathrm{n}-2)+\mathrm{a} 3 * \mathrm{x}(\mathrm{n}-3)+\mathrm{b} 1 * \mathrm{y}(\mathrm{n}-1)+\mathrm{b} 2 * \mathrm{y}(\mathrm{n}-2)+\mathrm{b} 3 * \mathrm{y}(\mathrm{n}-3) \\
S_{e, \Pi, e, \Pi}(f)=\sum_{m=0}^{N-1}\left[\sum_{k=0}^{N-1} s_{k} \cdot e^{-j 2 \pi \frac{k \cdot m}{N}}\right] \cdot \delta(f-m / \tau)
\end{array}
$$

Notons que c'est uniquement l'information de l'amplitude qui est calculée et affichée, la représentation fréquentielle $\mathrm{du}$ nombre d'échantillons peut se faire dans le domaine fréquentiel [0, $\mathrm{Fe}[$ ou de façon symétrique $]-\mathrm{Fe} / 2,+\mathrm{Fe} / 2]$. 


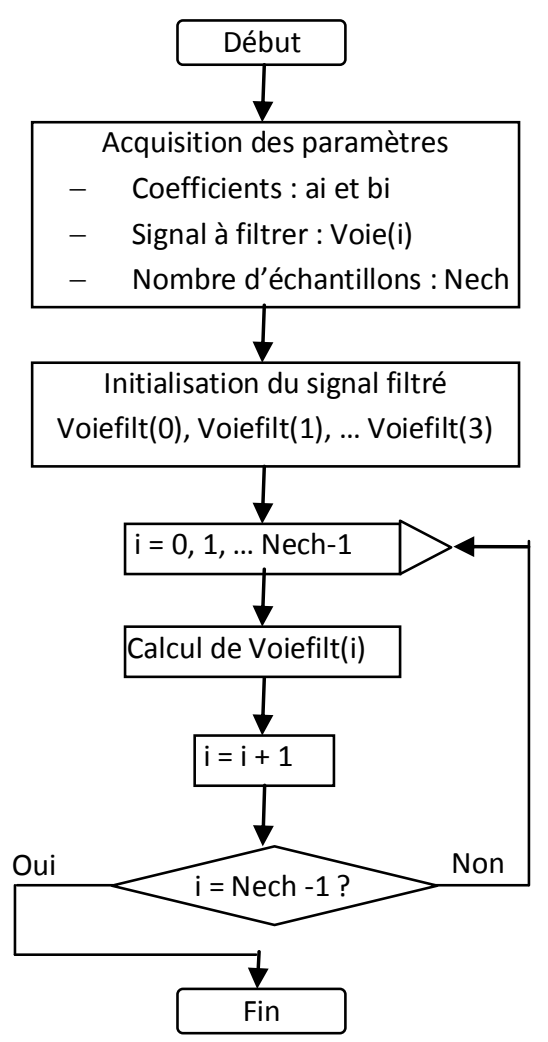

(a)

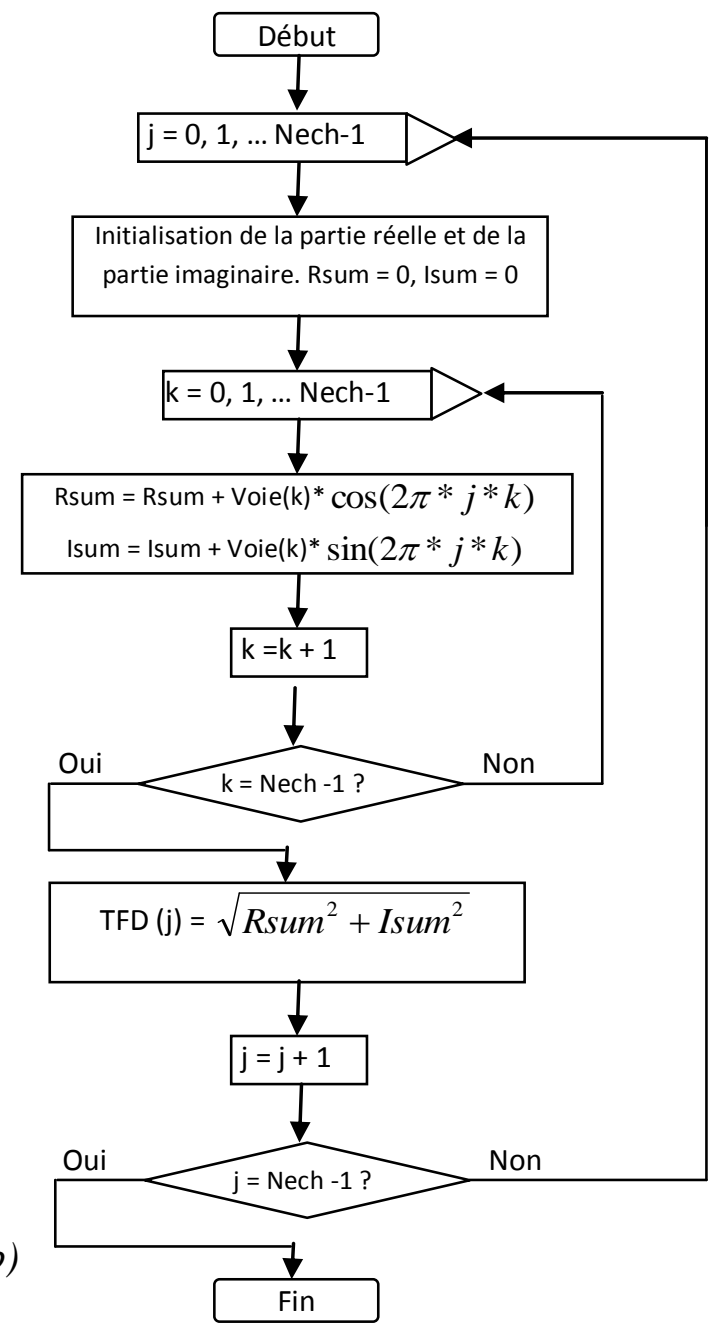

(b)

Figure 8. Organigramme des algorithmes : (a) du filtrage numérique, et (b) de la transformée de Fourier Discrète.

\subsubsection{Le module de sauvegarde des données}

Ce module permet de sauvegarder en fichiers d'extension.$M$ les données acquises par l'ordinateur exploitable dans MATLAB. Les principales lignes de code du programme utilisé ici sont issues de l'article de J. Mbihi [7].

\section{Manipulation du didacticiel}

La manipulation du didacticiel d'instrumentation proposé lors des travaux pratiques se passe en quatre principales étapes :

\subsection{Préparation des travaux pratiques}

- Etudier séparément grâce à la modularité de la chaine d'acquisition, le circuit électrique et la fonction de chaque carte ;

- Etudier les fonctions logicielles qui entrent dans la génération des ECG et la Conversion Analogique Numérique implantés dans la mémoire programme du microcontrôleur ;

- Etudier les principales procédures Visual Basic qui permettent de communiquer avec la carte d'acquisition, de reconstituer les données de 10 bits provenant du 
CAN, de retrouver la tension analogique originale, d'afficher l'allure de la tension, de filtrer, de calculer la TFD.

\subsection{Calibrage du Gain d'amplification et visualisation des courbes.}

- Connexion de la chaine d'acquisition complète au PC et mise sous tension ;

- Connexion aux entrées d'un amplificateur d'instrumentation du générateur d'ECG et générer le signal sinusoïdal destiné au calibrage du gain ;

- Lancement du logiciel installé dans l'ordinateur ;

- Estimation de la fréquence d'échantillonnage maximale de l'ordinateur en cliquant sur le bouton Fe $\max$ (Figure 9a);

- Calibrage du gain d'amplification de l'étage amplificateur d'instrumentation en cliquant sur le bouton Calibrage puis en jouant sur la résistance ajustable prévue à cet effet. Une fois le gain souhaité atteint, cliquer sur le bouton Quitter pour arrêter le calibrage (Figure 9c);

- Recommencer, si nécessaire, la manipulation pour les deux autres étages amplificateurs.

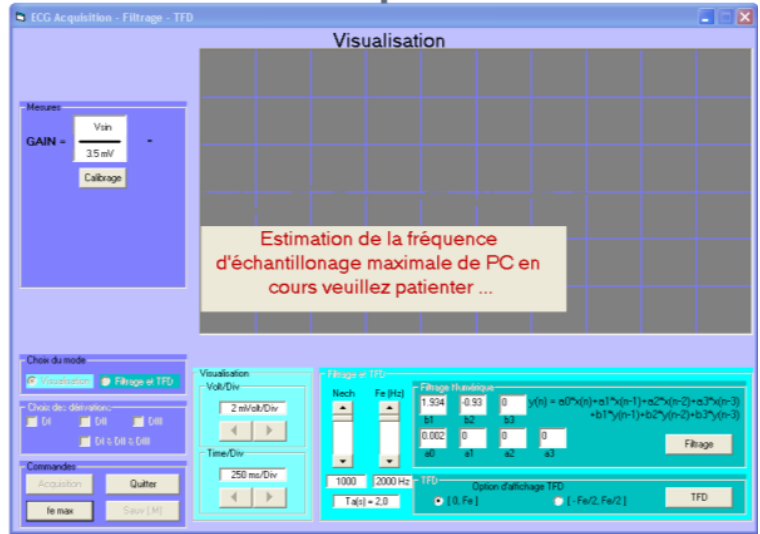

(a)

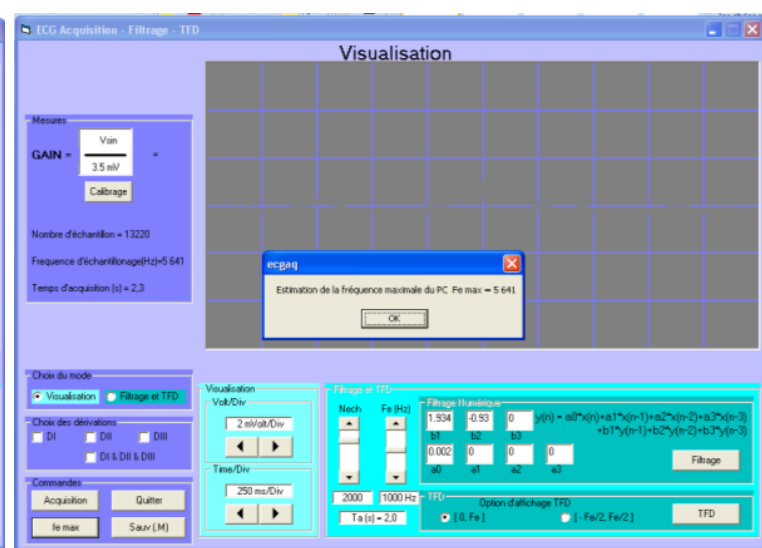

(b)

(c)

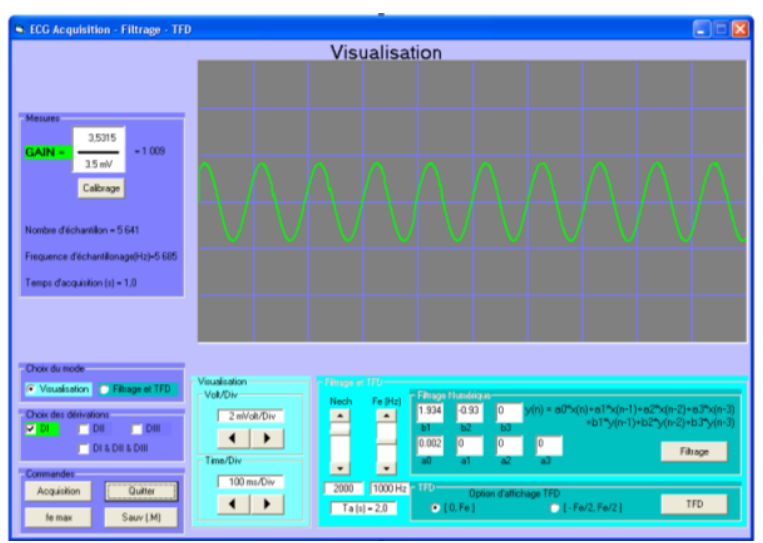

Figure 9 : Estimation de la fréquence d'échantillonnage maximale du PC (a) et (b), Calibrage du gain de l'amplificateur d'instrumentation de la voie sélectionnée (c). 


\subsection{Acquisition et visualisation des signaux.}

- Sélectionner sur le didacticiel le mode Visualisation (Mode sélectionné par défaut au démarrage) ;

- Placer les sondes aux extrémités des membres d'un sujet pour l'acquisition des trois dérivations DI, DII et DIII pour un ECG réel, ou connecter le générateur d'ECG;

- Sélectionner la ou les dérivations à visualiser ;

- Sélectionner les échelles des divisions du temps et de la tension en cliquant sur les boutons UpDown correspondants ;

- Cliquer sur le bouton Acquisition pour visualiser en temps réel l'allure des signaux choisis (Figure 10);

- Cliquer sur Stop pour arrêter l'acquisition et la visualisation en temps réel.

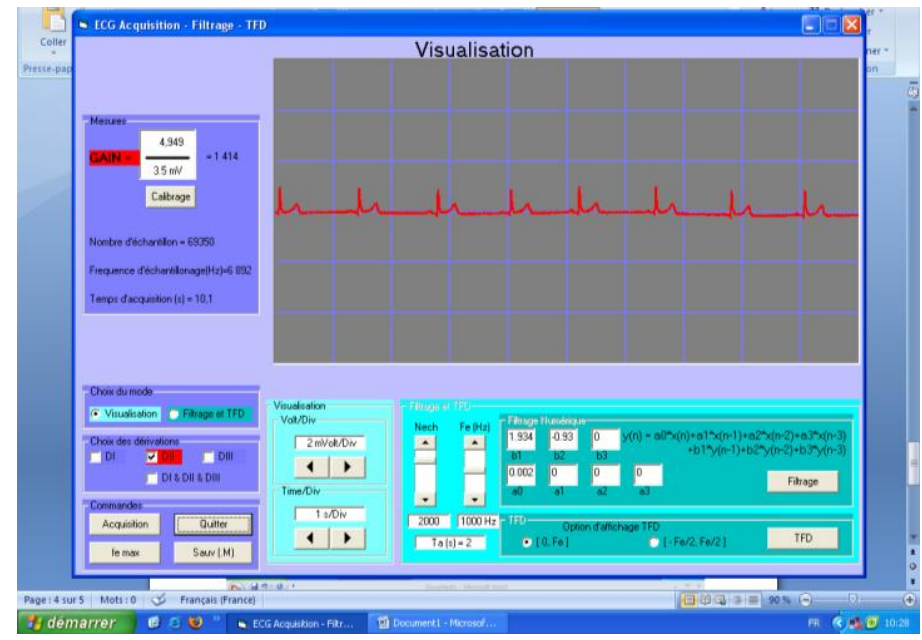

(a) Dérivation DII d'un signal ECG réel

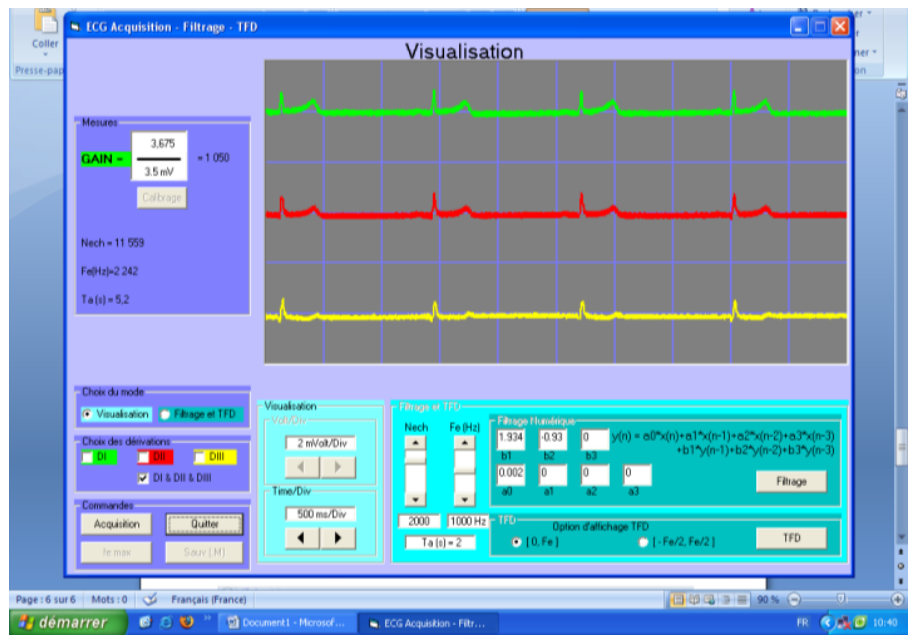

(b) Dérivations DI, DIl et DIII d'un signal ECG réel

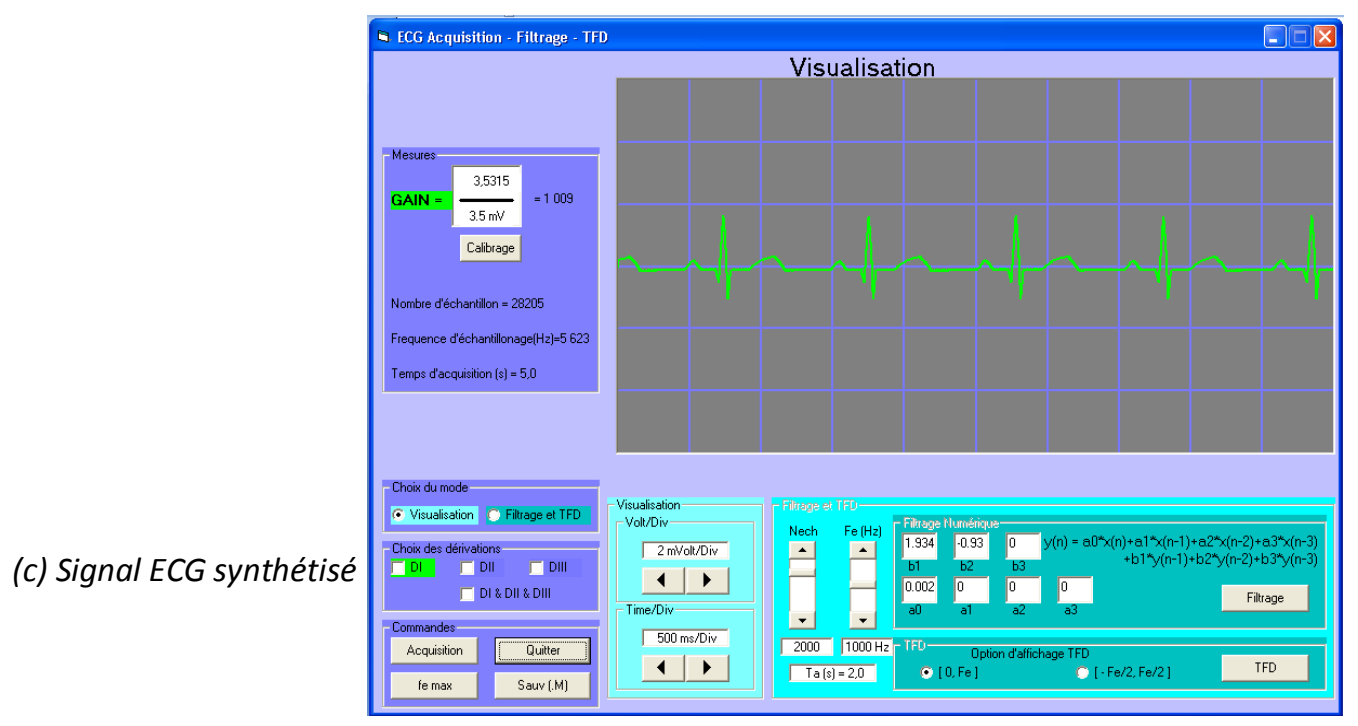

Figure 10 : Exemple d'acquisition d'un signal ECG réels et synthétisé 


\subsection{Filtrage et Transformée de Fourier Discrète.}

- Sélectionner le Mode Filtrage et TFD (Figure 11);

- Sélectionner la dérivation à traiter ;

- Fixer le nombre d'échantillon et la fréquence d'échantillonnage désiré en jouant respectivement sur les curseurs $\mathrm{Nech}$ et $\mathrm{Fe}(\mathrm{Hz})$;

- Cliquer sur le bouton Acquisition pour visualiser le nombre d'échantillon de la tension voulue ;

- Saisir les paramètres du filtre puis cliquer sur Filtrage ;

- Cliquer sur le bouton TFD pour afficher la Transformée de Fourier Discrète des signaux acquis et filtré ;

- Cliquer sur le bouton $\operatorname{Sauv}(. M)$ pour enregistrer les données du signal acquis dans un fichier d'extension $M$;

- Cliquer sur le bouton Quitter pour éventuellement fermer l'application.

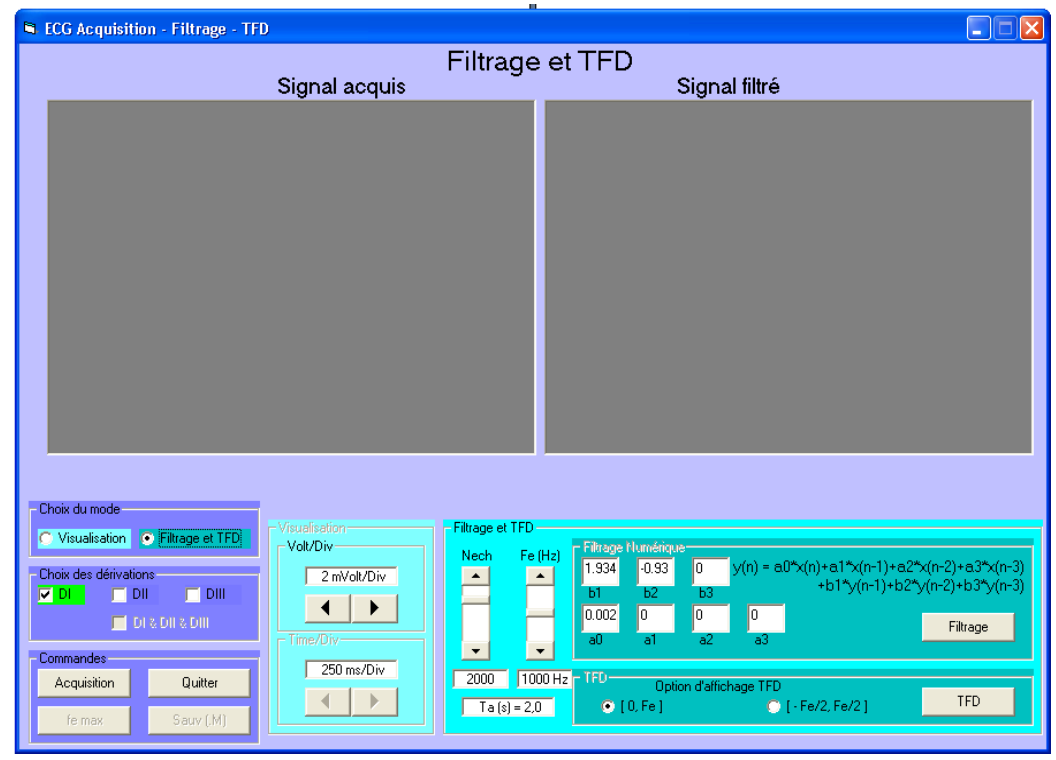

Figure 11 : Copie d'écran du logiciel d'instrumentation virtuel en mode Filtrage et TFD

\subsubsection{Exemple de filtrage du bruit de $50 \mathrm{~Hz}$}

Pour cet exemple, le générateur ECG fournis le signal ECG bruité avec le $50 \mathrm{~Hz}$ à une entrée de notre choix. La capture d'écran à la figure 12 représente à gauche le tracé de 2001 échantillons d'un signal bruité échantillonné à $400 \mathrm{~Hz}$, soit un temps d'acquisition d'environ 5secondes d'après la formule $T a=N e c h / F e$. Le tracé à droite est le signal filtré à l'aide d'un filtre numérique passe bas d'ordre 2 obtenu par équivalence de la dérivation de la façon suivante [6] :

$$
H(p)=\frac{\omega_{0}^{2}}{\omega_{0}^{2}+2 \omega_{0} m \cdot p+p^{2}} \stackrel{p \rightarrow \frac{1-z^{-1}}{T_{e}}}{\longrightarrow} H(z)=\frac{\omega_{0}^{2} T_{e}^{2}}{\left(1+2 \omega_{0} m T_{e}+\omega_{0}^{2} T_{e}^{2}\right)-2\left(1+\omega_{0} m T_{e}\right) \cdot z^{-1}+z^{-2}}
$$


à partir de la transmittance $H(z)$, le calcul de l'équation aux différences donne l'équation suivante : $\quad y(n)=a 0 * x(n)+b 1 * y(n-1)+b 2 * y(n-2)$.

Avec les valeurs des coefficients :

$$
a_{0}=\frac{\omega_{0}^{2} T_{e}^{2}}{1+2 \omega_{0} m T_{e}+\omega_{0}^{2} T_{e}^{2}}, b_{1}=\frac{2 \cdot\left(1+\omega_{0} m T_{e}\right)}{1+2 \omega_{0} m T_{e}+\omega_{0}^{2} T_{e}^{2}} \text { et } b_{2}=\frac{-1}{1+2 \omega_{0} m T_{e}+\omega_{0}^{2} T_{e}^{2}}
$$

L'application numérique pour une fréquence de coupure $\mathrm{F}_{0}=30 \mathrm{~Hz}$, le pas $\mathrm{Te}=0,0025 \mathrm{~s}(\mathrm{Fe}=$ $400 \mathrm{~Hz}$ ) et le coefficient d'amortissement $\mathrm{m}=0,7$ nous permet d'avoir les paramètres suivants : $\mathrm{a}_{0}=0,11792 \quad \mathrm{~b}_{1}=1,41364 \quad$ et $\quad \mathrm{b}_{2}=-0,53156$.

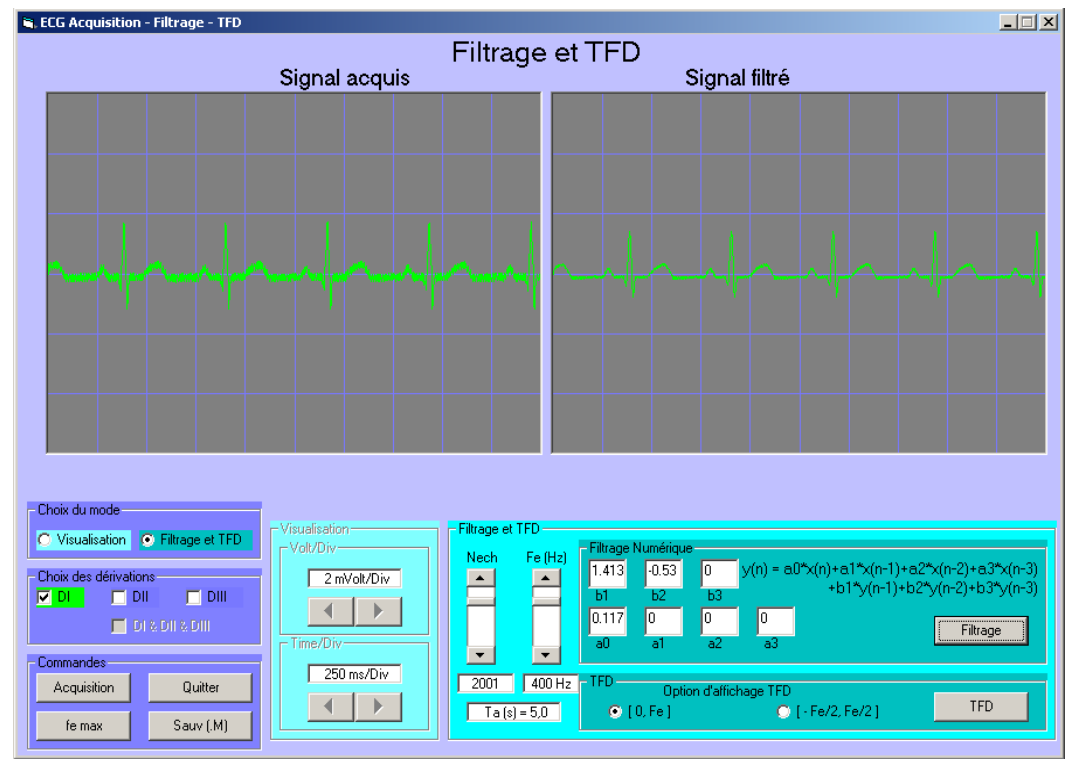

Figure 12 : Acquisition du signal bruité au 50Hz et Filtrage par un filtre numérique.

L'étape suivante consiste à visualiser le spectre d'amplitude des deux tensions selon la représentation souhaitée et apprécier l'influence du filtrage sur le signal original. (Figure 13)

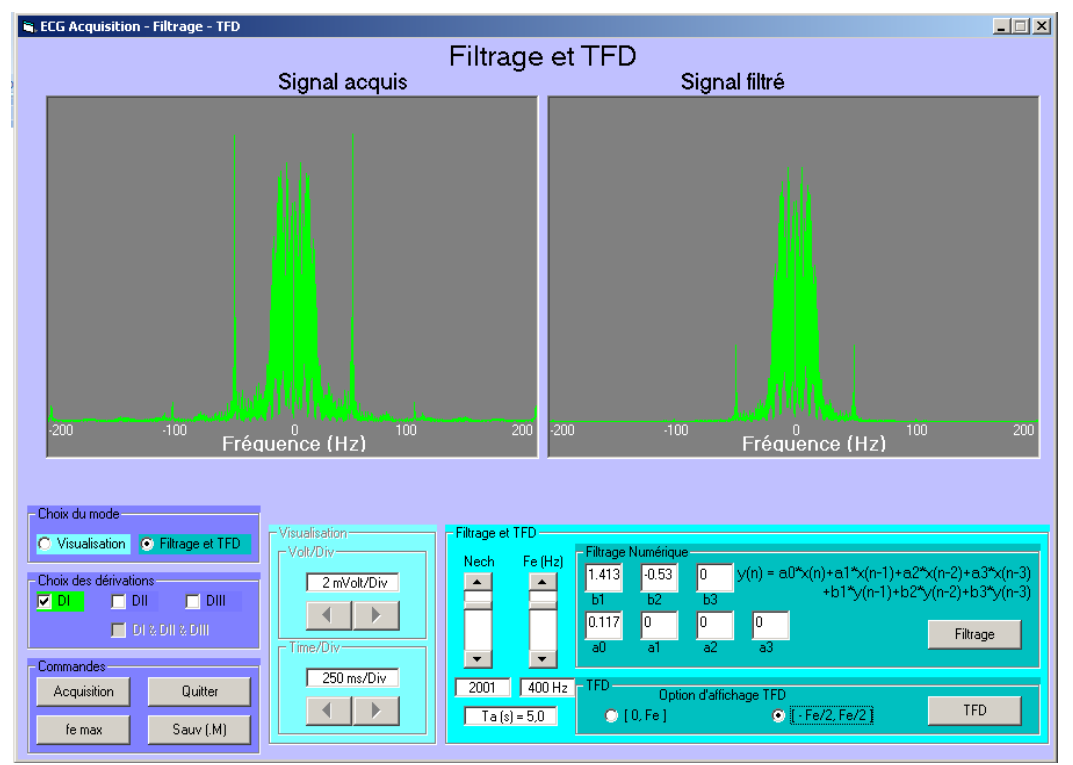


Figure 13 : Spectre d'amplitude de la tension échantillonnée acquise bruitée et filtrée.

Le passage à MATLAB se fait en sauvegardant le signal acquis et bruité. Le fichier Ecgacq.M obtenu de la figure 14 représente la matrice à trois colonnes $\left(n, n T_{e}, y(n)\right)$ et autant de lignes que de nombre d'échantillons du signal acquis.

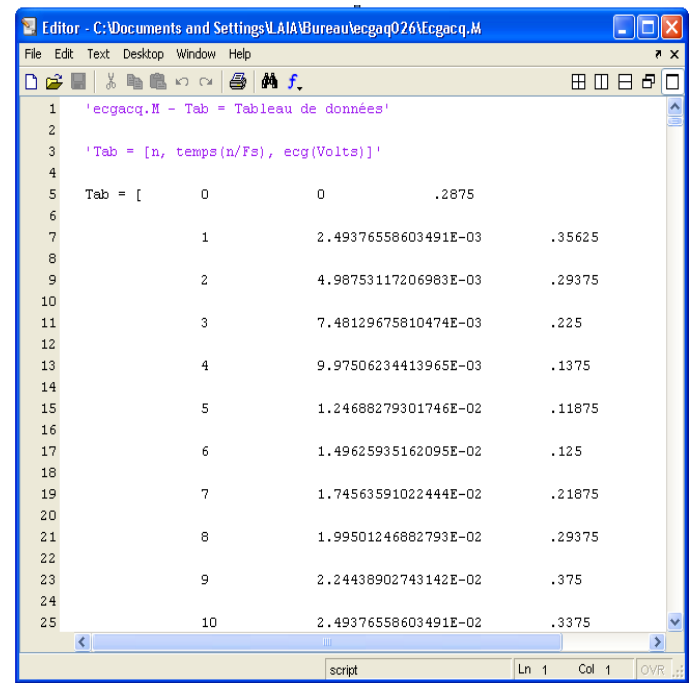

Figure 14 : Extrait du fichier de sauvegarde dans MATLAB.

Les données affichées et traitées sous MATLAB en utilisant un filtre avec les caractéristiques identiques à celui étudié dans le didacticiel nous donnent les résultats suivants.

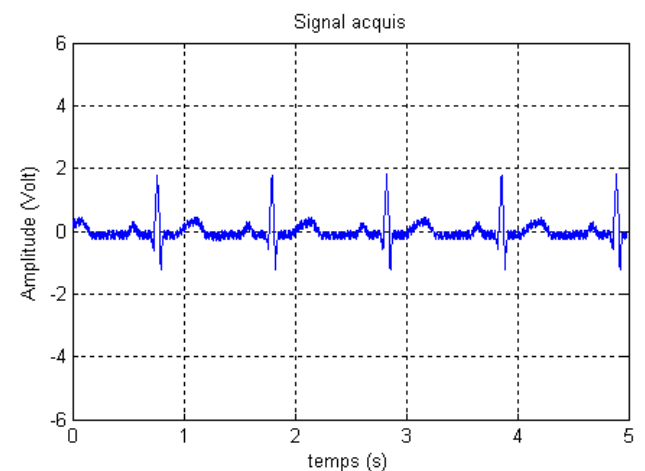

(a)
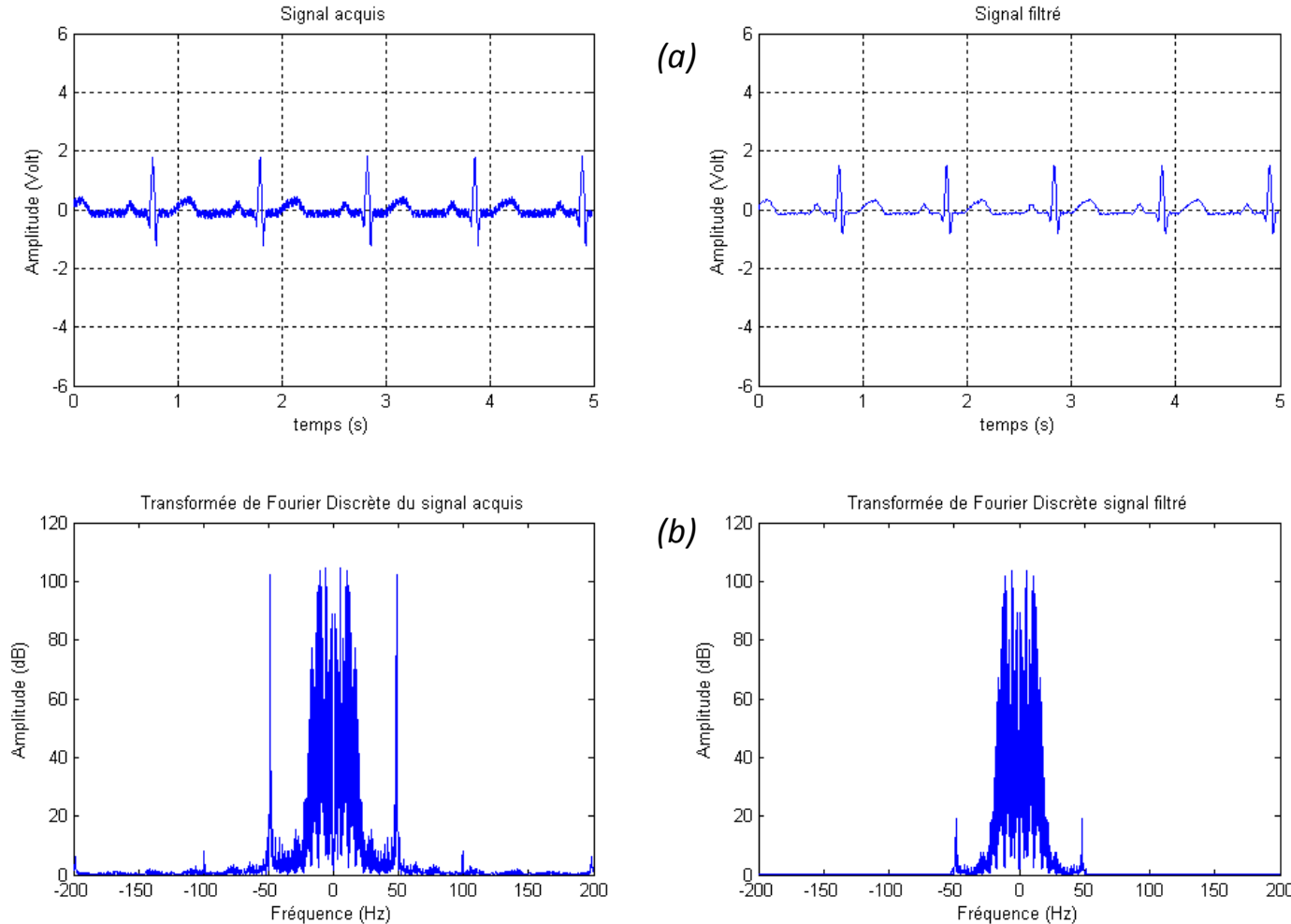

(b)

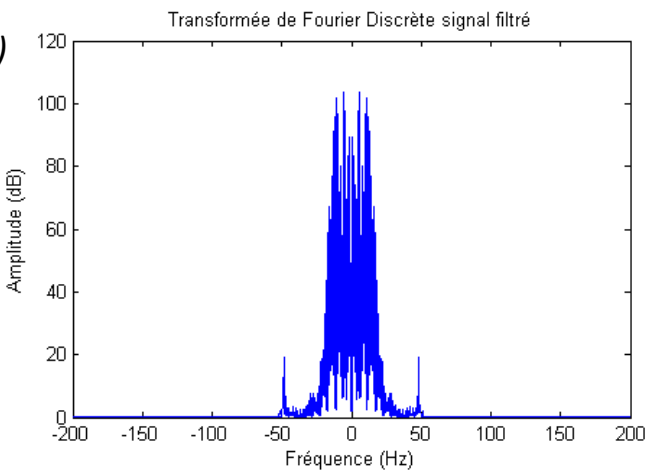

Figure 15 : Tracé sous MATLAB du signal acquis bruité et filtré dans le domaine (a) temporel et (b) fréquentiel 


\section{Conclusion}

Le didacticiel d'instrumentation virtuel présenté ici est une évolution certaine à l'IUT FV de Bandjoun dans l'enseignement pratique de l'instrumentation, de la programmation des systèmes, de l'acquisition des données et du traitement numériques des signaux en général et des signaux ECG en particulier. En associant uniquement la carte d'acquisition à un GBF de laboratoire il est possible, aux étudiants à l'aide du même logiciel, d'expérimenter le théorème de Shannon, de faire l'étude des caractéristiques fréquentielles des signaux sinusoïdaux, carrés ou triangulaires. La simplicité des circuits et le coût réduit de l'ensemble de la chaine d'acquisition nous permet d'étudier la possibilité d'une production en série pour en faire bénéficier une plus large communauté universitaire. Les perspectives seraient d'étendre les possibilités de la carte d'acquisition en augmentant le nombre d'entrée analogiques à 8 , ainsi il sera possible d'avoir les 12 dérivations standards d'un ECG. Cela nécessitera un transfert des bits plus rapidement du Microcontrôleur vers le port LPT du PC ou l'utilisation d'un port plus rapide comme le Port USB. Cette carte plus complète serait un outil important pour les chercheurs avec la possibilité de travailler sur des signaux réels, de développer la notion de vectocardiographie, d'implémenter des algorithmes pour la compression des signaux, la détection et la classification des battements cardiaques pathologiques.

\section{Références bibliographiques}

[1] V. MARKANDEY, " ECG Implementation on the TMS320VC5505 DSP Medical Development Kit (MDK) ", Application Report, Texas Instruments, July 2009.

[2] P. BAUCOUR, " L'Instrumentation Virtuelle Comme Outil Pédagogique ", Colloque sur l'Enseignement des Technologies et des Sciences de l'Information et des Systèmes (CETSIS), 25-27 Octobre 2005, Nancy.

[3] P. RAVIER, C. DUMEZ-VIOU, R. CANALS, " Traitements temps réel de signaux ECG : Un exemple de transfert recherche vers enseignement pour les systèmes embarqués", CETCIS, 25-27 Octobre 2005, Nancy.

[4] A. E. MARTINEZ, E. ROSSI, L. N. SIRI, " Microprocessor-based simulator of ECG signals ", $16^{\text {th }}$ Argentine Bioengineering Congress and the $5^{\text {th }}$ Conference of Clinical Engineering, Journal of Physics: Conference Series 90, 2007.

[5] J. MBIHI, " Informatique et Automation : Automatismes Programmable Contrôlés par Ordinateur ", Editions Publibook Universités, Paris, 2006.

[6] François COTTET, " Traitement du Signal - Aide mémoire", Editions DUNOD, Paris, 2005.

[7] J. MBIHI, "Contribution à l'étude et au prototypage d'un banc d'essai didactique flexible d'instrumentation virtuelle et d'asservissement par ordinateur ", Journal sur l'enseignement des sciences et des techniques de l'information et des systèmes, J3EA - Vol. 9 No 1 (2010), EDP Sciences. 


\section{Site internet}

[int1] http://logix4u.net

\section{Biographie des auteurs}

TCHIMMOUE Gaby Eric est titulaire du diplôme de Professeur d'Enseignement Technique (DIPET) de $2^{\mathrm{e}}$ grade en EEA obtenu à l'ENSET de Douala au Cameroun. Il est également titulaire d'un DEA en électronique obtenu à l'université de Yaoundé I. Il poursuit une thèse, dans le domaine de l'électronique biomédicale, au Laboratoire d'Electronique et du Traitement du Signal (LETS) de l'Ecole Nationale Supérieure Polytechnique (ENSP) de Yaoundé sous la direction de M. Kamdem jean et M. Nko'o Amvene S. Il est actuellement Attaché d'Enseignement et de la Recherche (ATER) à l'IUT Fotso-Victor de Bandjoun.

KAMDEM KOUOKAM Jean est Docteur d'Etat en électronique et enseignant chercheur à l'ENSP où il est responsable des unités d'enseignements de l'électronique analogique et de la microélectronique. Il développe ses recherches au Laboratoire d'Electronique et du Traitement du Signal (LETS) dans le domaine de la microélectronique et de l'électronique biomédicale.

NKO'O AMVENE Samuel est Professeur et Vice-Doyen chargé de la programmation et du suivi des activités académiques à la Faculté de Médecine et des Sciences Biomédicales de l'Université de Yaoundé I. Il est également chef du service Radiologie/Imagerie Médicale du Centre Hospitalier et Universitaire de Yaoundé (CHUY). 